\title{
Uso de Google Classroom como repositorio de robótica práctica: PieroAcademy
}

\author{
Antonio J. Muñoz-Ramírez, Jesús M. Gómez-de-Gabriel, \\ Juan M. Gandarias, José Cárdenas, Jaime Molina, Anthony Mandow \\ Universidad de Málaga, Dto. de Ingeniería de Sistemas y Automática \\ aj@uma.es
}

\section{Resumen}

En el desarrollo de prácticas de laboratorio en algunas asignaturas del área de ingeniería de sistemas y automática, los alumnos recaban información relativa a equipos y componentes de diversas fuentes externas. Los resultados de su trabajo, que en muchos casos son de gran calidad, pasan al olvido una vez evaluados. Con el objetivo de poner en valor y mejorar el aprovechamiento de dichos trabajos, se propone la creación de un repositorio controlado de información, donde los estudiantes puedan recopilar parte del material necesario para la realización de sus trabajos, ejemplos de ayuda, $y$ tutoriales realizados por otros estudiantes; pero además contribuir a ampliar la información mediante sus propias experiencias. En la implementación del repositorio, se hace uso de las herramientas proporcionadas por la $G$ Suite for Education (GSFE), siendo el núcleo, la herramienta denominada Google Classroom. En este trabajo se describe la implantación y experiencia con este sistema como medio para gestionar un repositorio organizado donde sus denominadas "clases", toman contenido de unidades temáticas; con la versatilidad de su acceso desde dispositivos móviles y la capacidad de reutilización en asignaturas reales.

Palabras clave: Google Classroom, GAFE, GS$\mathrm{FE}$, repositorio robótica, tutoriales, aprendizaje semipresencial

\section{INTRODUCCIÓN}

Las nuevas tendencias en la enseñanza de la ingeniería vienen ligadas a conceptos como aprendizaje colaborativo (AC), aprendizaje basado en problemas/proyectos (ABP), aprendizaje autónomo, explicar para aprender, capacitación transversal, resultados de aprendizaje, fomentar el espíritu crítico, rúbrica, autoevaluación, evaluación entre pares, competencias profesionales. La incorporación de estos conceptos en la formación universitaria, aun incluso de forma parcial, forma parte de una metodología integradora que aproxima al estudiante a su futura vida profesional. Profesores de la Universidad de Málaga, tras varios proyectos de innovación educativa, ya emplean esta metodología docente en sus clases $[1,2,3,4,5]$.

El ámbito de las asignaturas objeto de este trabajo corresponde al aprendizaje de conceptos de control y robótica a partir de su aplicación práctica, principalmente con dispositivos desarrollados por los autores en proyectos de innovación educativa de la Universidad de Málaga PIE15-180 y PIE13-134. Estos dispositivos son el robot móvil PIERO y el UMA Haptic (ver figura 1), los cuales se hacen protagonistas de las asignaturas, donde las prácticas toman ahora una mayor relevancia. Con la intención de acercar al alumno al mundo profesional, a las asignaturas se le aplica una orientación de trabajo en equipo y aprendizaje basado en proyectos/problemas; y un enfoque de desarrollo de soluciones basado en modelos mediante lenguajes de modelado de dominio específico.
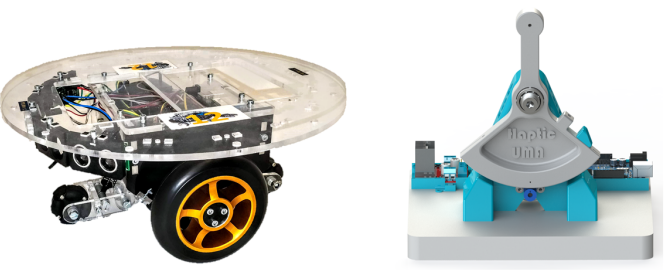

Figura 1: Dispositivos desarrollados para las clases: plataforma robótica móvil PIERO (4 ${ }^{\mathrm{a}}$ versión) y el UMA Haptic

\section{OBJETIVOS}

El objeto del actual proyecto de innovación docente, que ha dado pie a este trabajo, es la creación de un soporte en-linea (ver figura 2) para la realización de prácticas docentes basadas en $\mathrm{AC}$ y $\mathrm{ABP}$, que sirva como fuente de información previa al trabajo de los alumnos, y al mismo tiempo permita recoger y catalogar los resultados obtenidos para sucesivos trabajos. De esta forma el material didáctico para el aprendizaje autónomo se incrementará mediante la selección revisada de 


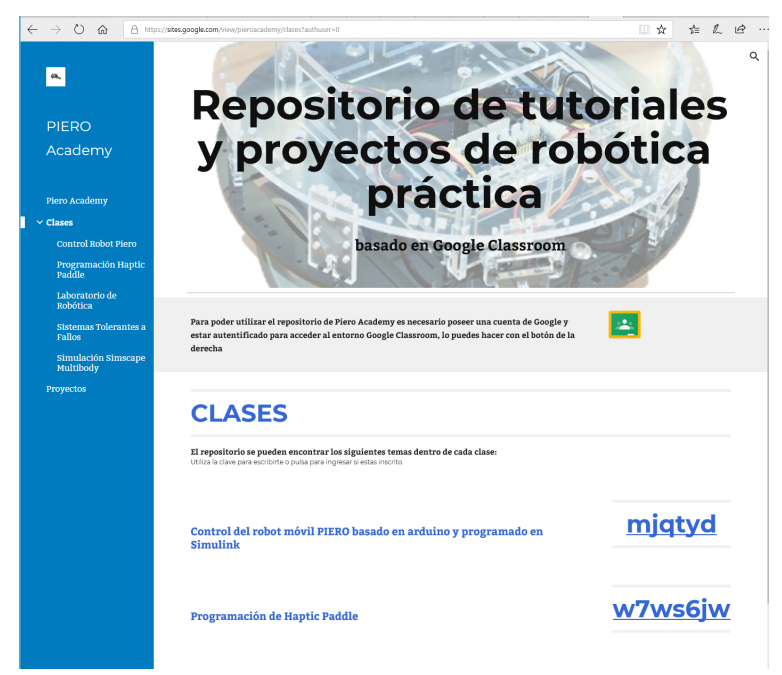

Figura 2: Vista PC de la página web del repositorio PieroAcademy.net, creada con Google Sites, donde se indican las clases disponibles y su código de acceso

la producción de los estudiantes. Asimismo, estos contenidos estarán abiertos para su libre disposición por la comunidad educativa. Se obtendrá una herramienta para el desarrollo de la autonomía y la autorregulación del aprendizaje del alumno, a la vez que se potencia el uso del trabajo cooperativo.

Se trata, por tanto, de mejorar la manera en la que se utiliza la información en las prácticas de robótica, mecatrónica y control, para que los alumnos sean más eficientes y su trabajo sea compartido, reutilizado y publicado. Además, este sistema permite dotar a los estudiantes de evidencias de las competencias profesionales que han adquirido, enlazando sus aportaciones al portafolios de su currículum.

La robótica ha pasado de impartirse tan solo en titulaciones universitarias especializadas a enseñarse en todos los niveles educativos [6]. En este este sentido, el acceso libre al repositorio permitiría aumentar el número de usuarios al mismo tiempo que captar a futuros estudiantes, marcándose como objetivo secundario.

Las premisas o características a cumplir por este repositorio para los objetivos propuestos son:

- Multiplataforma: posibilidad de utilización tanto en el ordenador como en los móviles o tabletas. Si bien la visualización y ejecución de los códigos puede limitarse a un ordenador, la documentación y los vídeos podrán incrementar su visibilidad a través de dispositivos móviles.

- Acceso abierto: disponibilidad de un registro de acceso (es de interés conocer quién la usa).
- Modularidad: facilitar la descomposición y almacenamiento en unidades temáticas.

- Puntuación: posibilidad de calificar la calidad de los módulos.

- Alojamiento sencillo, instalación y configuración no complejas, así como gran capacidad de espacio y ancho de banda.

- Gestión simple compartida: capacidad para varios administradores a fin de no aumentar la carga del profesorado ni focalizarla en un solo individuo.

- Coste: a tener en cuenta la menor carga presupuestaria posible.

Hay que hacer hincapié, en el hecho de que no se busca un LMS (Learning Management System), sino una solución mucho más simple que permita compartir archivos. Teniendo en cuenta las características anteriores, se evaluaron soluciones basadas en Wordpress, Joomla, Mahara, y Google Classroom, siendo esta última solución la adoptada por aventajar a las demás en la mayoría de apartados, pero sobre todo, por su facilidad de implementación, completada por el resto de herramientas de la $G$ Suite for Education (GSFE).

\section{Implementación de GSFE en PieroAcademy}

\subsection{Antecedentes}

Muchas universidades gestionan un LMS o campus virtual propio que facilita el aprendizaje en línea de sus estudiantes. Además, es común disponer de acuerdos con Google que permitan incoporar GSFE en la institución. Tal es el caso de la Universidad de Málaga(UMA), donde la propia cuenta corporativa facilita el acceso a las herramientas de GSFE. Estas incluyen el correo electrónico basado en interfaz Google (Gmail o Inbox), Google Calendar y Google Drive, herramientas para edición de documentos (Google Docs), hojas de cálculo (Sheets) y elaboración de presentaciones (Slides). La herramienta Google Forms (para formularios o cuestionarios) ofrece interés por su versatilidad en la incorporación de multimedia en las preguntas y un plus de seguridad al ser el profesor el único responsable de su custodia.

Google Classroom apareció en 2014 [7] como plataforma de blended learning (aprendizaje semipresencial), inicialmente solo disponible para centros educativos con cuentas corporativas. En 2017 se abrió a las cuentas no corporativas de GSFE [8], y con la posibilidad de acceso a las clases de Google 
Classroom corporativo con cuentas ajenas al propio dominio y viceversa. Sin embargo, en la UMA el acceso de Classroom se mantiene exclusivo para las cuentas corporativas, impidiendo también que puedan acceder a clases de Classroom creadas por dominios distintos de uma.es. Por tanto, es necesaria la creación de un dominio propio para posibilitar el acceso libre, en este caso PieroAcademy.net, y garantizar el control de los permisos de administración de GSFE, utilizando como guía el tutorial disponible en [9].

Las herramientas de la G Suite for Education (GSFE), conocida anteriormente por Google Apps for Educaction (GAFE) han sido empleadas con éxito en la educación superior, con Docs [10], Drive $[11,12]$, Sites [13], o como GAFE $[14,15,16]$. Al principio, tan solo era un Sistema de Gestión de Contenidos - CMS (Content Management System), que incluye la creación y administración de los contenidos de una página Web, pero con la incorporación de Classroom, GSFE posee también un Sistema de Gestión de Aprendizaje - LMS que automatiza las acciones de formación. Básicamente Classroom pone orden en los permisos de DRIVE a la hora de compartir los archivos de la clase, realizándose esta tarea de forma automática y alternando la propiedad de los archivos entre el alumno y profesor en función de la fase de envío o revisión que se encuentre el archivo. Por otra parte, los beneficios de la utilización de Classroom en la educación superior quedan demostrados en $[17,18]$.

Si bien las intenciones de la compañía Google ponen énfasis en el uso de GSFE en la educación en niveles inferiores a la educación superior como herramienta fundamental [19], en una apuesta clara por crear futuros usuarios/clientes fieles, para la universidad la considera como una herramienta complementaria.

Dos elementos que destacan de Classroom frente a otros LMS son su diseño pensado en los smartphones más que en el PC (posee aplicaciones para android e IOs), y su presentación de tareas, marcada por un tablón estilo Facebook, es decir orientada cronológicamente a diferencia de la organización por temas de otros LMS como Moodle.

El repositorio PieroAcademy.Net podía haber utilizado tan solo las herramientas CMS, no obstante, el uso de Classroom (LMS), mediante la aplicación móvil, permite un registro de su uso, y una utilización en asignaturas reales prescindiendo o no del actual LMS de la UMA. la inscripción a una clase de Classroom es mediante registro conociendo la clave, o mediante invitación directa de la cuenta por parte de un profesor. Por tanto, presenta un problema para el acceso en abierto sin supervisión.
La forma mediante la que se evita consiste en el uso de una página web que publique la clase junto con su clave (véase Figura 2).

\subsection{Estructura del Repositorio}

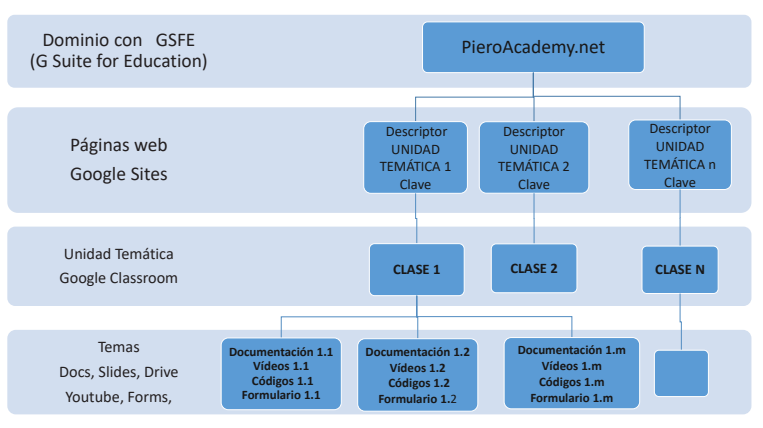

Figura 3: Estructura del repositorio PieroAcademy.net

Una visión general de la estructura del repositorio se puede observar en la figura 3, donde aparecen:

- Como raíz el dominio "PieroAcademy.Net"que ha sido utilizado en la implementación de GSFE con verificación de dominio. Sobre dicho dominio se ha creado tanto la página web del dominio residente en Sites, pero redireccionada desde pieroacademy.net.

- Las páginas dedicadas a describir el contenido de cada una de las unidades temáticas que forman parte del repositorio. La página deberá contener la clave de matriculación de la "CLASE", en donde se ha implementado la unidad temática.

- Las unidades temáticas (unidad de temas afines) implementadas como clases. La visión global del repositorio se mantiene gracias a empleo de una cuenta general denominada "pieroacademy", la cual es utilizada para la creación de cada clase; siendo por ello la cuenta propietaria de todas las clases del repositorio, y gestora de las clases junto con las cuentas de los profesores invitados.

- Los temas o componentes del tema, formados por agrupaciones de (documentación, vídeo, códigos, formulario), pudiendo según el caso prescindir de algunos de ellos.

\section{LAS UNIDADES TEMÁTICAS}

Las unidades temáticas representan el conjunto de temas o elementos de temática afín implementados con Google Classroom como una clase cuyo propietario es la cuenta pieroacademy. En la figura 
4 aparecen cuatro unidades temáticas en el panel web del PC de Google Classroom. Una vista rápida sobre Classroom se puede obtener en [20, 21], y una guía de creación de clases en $[22,23]$.
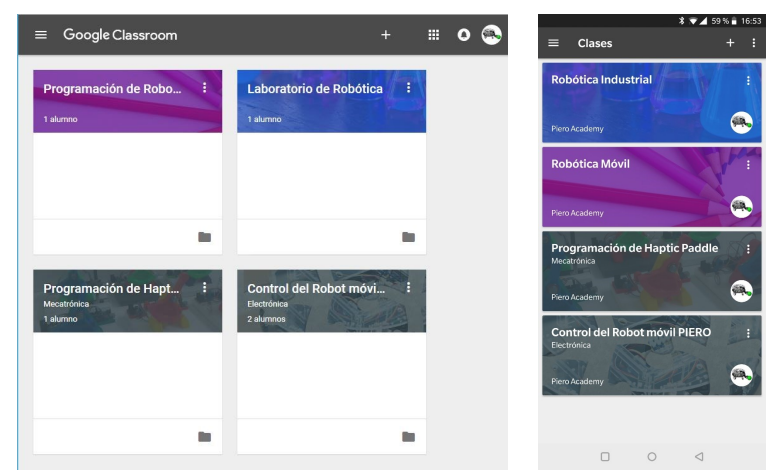

Figura 4: Vista del profesor en PC(izda.) y alumno en la App Classroom (dcha.) de unidades temáticas implementadas en Classroom.

Las Clases de Classroom poseen tres secciones, que se tratarán a continuación.

\section{- NOVEDADES / FLUJO DE ACTIVIDA-} DES: denominadas así según se acceda por web o móvil, similar al tablón de Facebook, aparecen los elementos o bloques ordenados cronológicamente, admitiendo una agregación por temas. Para el uso de repositorio se ha configurado que los alumnos puedan también publicar y realizar comentarios entre ellos. De está forma se consiguen contribuciones al repositorio de forma externa.

- AlumNos / COMPAÑEROS DE CLASE: según vista alumno o profesor. Es donde aparece la clave del curso, los alumnos inscritos, y la inscripción manual. También tiene su utilidad en el seguimiento individualizados de los alumnos, más valido para una asignatura que para el repositorio. Los alumnos tan solo pueden ver la identificación de sus compañeros de clase.

- INFORMACIÓN: donde se encuentra el contenido estático de la unidad temática, también los enlaces al Drive y Calendar, y la lista de profesores con su invitación.

La organización de la unidad temática o clase, puede darse en base a entradas en bloque y temas. En la figura 5 se puede observar la sección de flujo de actividades, el contenido de un bloque y la sección de información de la App android Classroom en la cuenta de estudiante. Los bloques están formados por documentación en formato pdf, documentos (Docs) o presentaciones (slides), vídeos, archivos comprimidos con códigos,

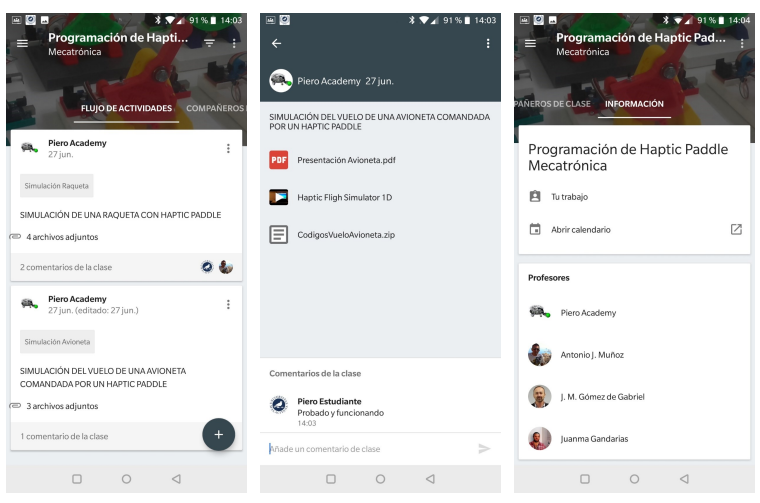

Figura 5: Vista de estudiante en la App Classroom del "Flujo de actividades", del contenido de un bloque, y de la sección de "información" de la unidad temática "Programación de Haptic Paddle".

etc. Aunque tradicionalmente como slides se usa el formato Powerpoint, si las presentaciones son estáticas, es mejor pasar a pdf para optimizar su visualización en el móvil; si posee transición y/o animaciones, entonces es conveniente convertirla a Slides para garantizar que sólo puede ser visualizada y no modificada.

El contenido de las unidades temáticas puede organizarse por temas dentro de la vista de flujo de actividades (ordenación cronológica) con contenido dinámico en el tiempo, o bien en la sección de información con contenido estático siempre activo. Por otra parte, se tienen dos tipos de unidades temáticas: la formada por proyectos únicos y la formada por aportaciones similares de distintos autores. Para las aportaciones repetitivas se ha creado un sistema de puntuaciones basado en un formulario con el fin de establecer una clasificación de la mejor aportación de cada temática. En la figura 6 se muestra una entrada de formulario para puntuación y una gráfica de puntuación global obtenida a partir del formulación con Sheets.
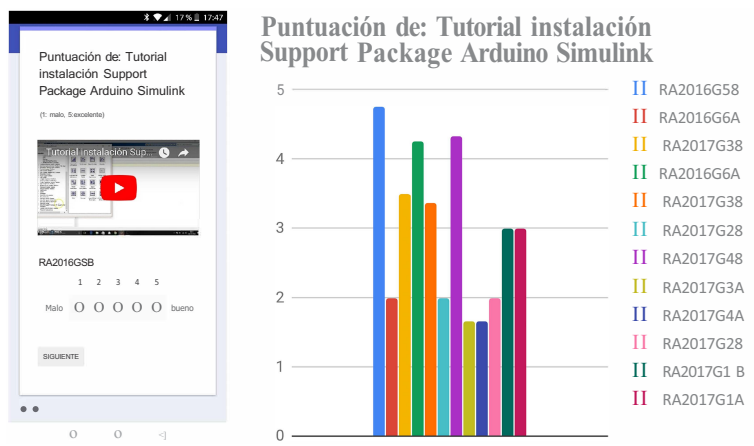

Figura 6: formulario de puntuación de un tutorial y resultado global de las puntuaciones. 


\section{ORGANIZACIÓN DE VIDEOS EN PIEROACADEMY.NET}

El hospedaje de los vídeos y su organización es un elemento clave en implementación del repositorio. La visualización de vídeos de forma masiva en alta resolución supone un consumo de ancho de banda difícil de mantener económicamente de forma privada, tampoco hay bastante soporte corporativo en la universidad para ello, así que la única vía razonable es recurrir otra vez a hosts externos, entre ellos los de Google. Dado que ya se está usando GSFE existen dos posibilidades: almacenamiento en Google Drive o utilizar Youtube. El almacenamiento de vídeos en Drive tiene dos inconvenientes que la descartaron: los vídeos se guardan tal y como se graban (tamaños enormes), y el segundo es el bajo ancho de banda que ofrece Drive, que sobre todo, en archivos grandes de vídeo oscila sin control de forma desesperante. Por otra parte, el almacenamiento de vídeos en Youtube posee compresión y optimización del vídeo de forma automática (incluso con correcciones de calidad), y ofrece un ancho de banda elevado que garantiza la correcta visualización del vídeo; por contra tiene otras dos dificultades, pero esta vez salvables:

- la administración múltiple, es decir, que los profesores puedan subir vídeos desde sus cuentas. Esto se consigue creando las denominadas «cuentas de marca» y dando permisos de administración a las cuentas de los profesores. En la figura 7 se muestra un diagrama de la organización realizada para PieroAcademy.Net.

- la organización temática, es decir poseer cierta clasificación temática de los videos, ello se consigue mediante la creación de los canales asociados a las cuentas de marca.

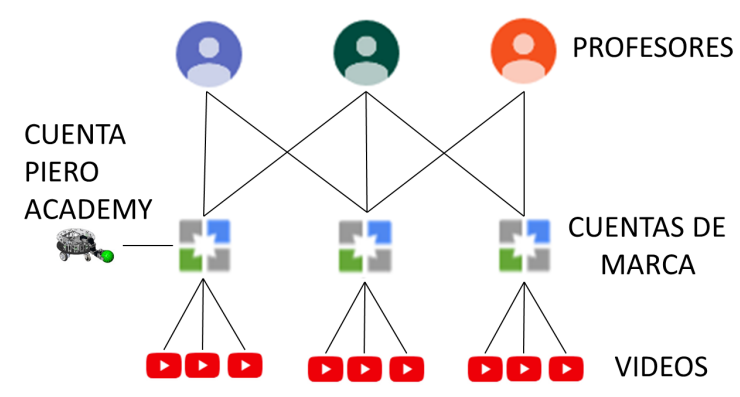

Figura 7: Organización temática de vídeos en Youtube con cuentas de marca, y desde múltiples proveedores (cuentas personales de los profesores)

El servicio de Youtube no se encuentra en la GS$\mathrm{FE}$, aunque sus videos poseen mayor integración que los de Drive (los vídeos de Youtube poseen previsualización en Classroom). Por lo tanto se recurre a crear una cuenta estándar de Gmail, como la propietaria de todas las «cuentas de marca». Las cuentas de marca deben ser verificadas por teléfono estando entre 4 y 5 por año y teléfono. En la figura 8 se muestran algunos de los canales creados para PieroAcademy.

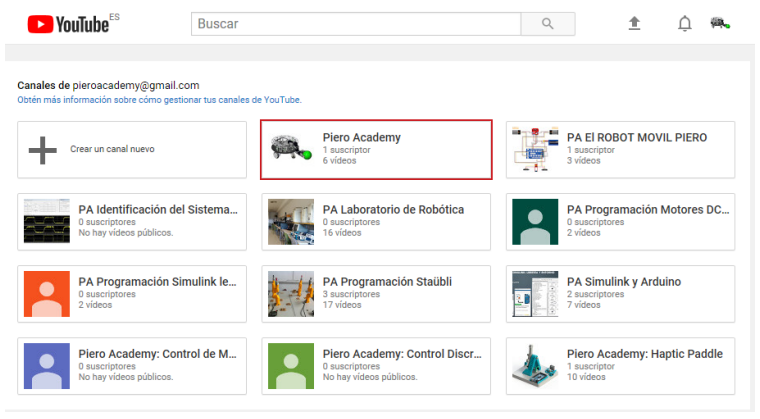

Figura 8: Canales de Youtube creados como cuentas de marca, para permitir la subida y administración desde las cuentas de los profesores.

\section{DISCUSIÓN Y CONCLUSIONES}

El uso de móvil como dispositivo de aprendizaje se está imponiendo entre la comunidad educativa desde edades tempranas, en especial ahora con Google Classroom, que pese a llevar poco tiempo está batiendo a soluciones consolidadas por ser complejas y poco optimizadas hacia los smartphones. Con la utilización de GSFE para la implementación de PieroAcademy.net, se ha dotado al repositorio de una interfaz amigable para smartphones, con capacidades CMS y LMS. De igual forma se ha creado una estructura para la utilización de la plataforma Youtube de forma conjunta y organizada por parte de los profesores. Por contra se tiene como principal inconveniente la dependencia de las decisiones de Google sobre esta plataforma, sobre su gratuidad, continuidad, o del uso que se hace de la información que pasa por sus servidores.

En esta primera fase de PieroAcademy, es en donde se ha diseñado e implementado una infraestructura para crear un repositorio para las asignaturas de robótica, control y mecatrónica, con capacidades de puntuación, de crecimiento, y de gestión que cumple con los objetivos marcados. Una vez dotado del contenido básico, se pasará a la fase de utilización durante el próximo curso 2018-19 en las asignaturas afines al repositorio y se extraerán resultados de la interacción de los estudiantes con él. Asimismo, los materiales elaborados por los estudiantes podrán incorporarse al repositorio para 
aumentar la base de conocimientos y experiencias sobre las diferentes asignaturas, manteniéndolas en evolución y actualizadas.

Como características pendientes de implementar, se encuentran la utilización de plataformas de control de versiones de código (tipo git o github), la incorporación de referencias bibliográficas en el repositorio vía Mendeley, la inclusión en los bloques de elementos de "gamificación"(tipo Kahoot), y por último la publicación de proyectos en Matlab Central, dado que matlab/simulink es el lenguaje de modelado y programación utilizado mayoritariamente, incrementando de esta forma la visibilidad del repositorio.

\section{Agradecimientos}

Este proyecto ha sido parcialmente financiado por el proyecto de Innovación educativa de la Universidad de Málaga PIE 17-118.

\section{English summary}

\section{Using Google Classroom as a reposi- tory of practical robotics: PieroAca- demy}

\section{Abstract}

In developing laboratory practices in some courses related to systems engineering and automation, students are expected to gather information on equipment and components from different external sources. However, the results of their research, which in many cases are of high quality, are forgotten once evaluated. In order to value and improve the use of these works, we propose the creation of a controlled repository of information where students can collect material required to carry out their labwork, examples, and tutorials made by other students, but also contribute to expand the available information through their own experiences. The implementation of the repository is based on tools recently provided by the $G$ Suite for Education (GSFE), especially Google Classroom. This paper describes the development and experience with this system as a means to manage an organized repository where the so-called "Classes"take content from thematic units. The experience has shown advantages regarding versatility in the access from mobile devices and the capacity of reuse in real courses.

Keywords: Google Classroom, GAFE, GSFE, robotics repository, tutorials, blend learning.

\section{Referencias}

[1] J. E. Gil Lozano, A. J. Muñoz Ramírez, V L Torres, and J M Gomez. Uso de Simulink y Arduino para prácticas de robótica. In Jornadas de Automática, pages 3-5, Valencia, Spain, 2014. Comité Español de Automática (CEA-IFAC).

[2] Antonio J Muñoz-Ramírez, J Jesús Fernández-Lozano, and $\mathrm{J}$ Manuel. Ingeniería Basada en Modelos en Prácticas de Robótica. In Jornadas de Automática, pages 624-630, Bilbao, 2015. Comité Español de Automática de la IFAC (CEA-IFAC).

[3] Antonio J. Muñoz-Ramírez and J.M. Gómezde Gabriel. Modelar o programar en prácticas de robótica. In Jornadas de Automática, pages 1-7, Madrid, 2016. Comité Español de Automática (CEA-IFAC).

[4] Juan M Gandarias, Antonio J Muñoz, and Jesús M Gómez-de Gabriel. Uso del Haptic Paddle con aprendizaje basado en proyectos. In XXXVIII Jornadas de Automática, pages 451-456, Gijón, 2017. Comité Español de Automática (CEA-IFAC).

[5] Antonio J. Muñoz-Ramírez and Jesús M. Gomez-de Gabriel. Experiencia docente en Automática empleando un robot móvil como elemento motivador de metodologías activas. In Indotec V- $5^{\circ}$ International Conference on Educational Innovation in Technical Careers, pages 77-82, Granada, 2017.

[6] Instituto Nacional de Tecnologías Educativas y de Formación del Profesorado (INTEF). Programación, robótica y pensamiento computacional en el aula. Situación en España, enero 2018. Technical report, 2018.

[7] Zach Yeskel. More teaching, Less tech-ing: Google Classroom Launches Today, 2014.

[8] Gene Ressler. Google Classroom: Now open to even more learners, 2017.

[9] Juan Carlos Guerra. Tutorial de Google Classroom - Gestión en consola de GSuite, 2017. 
[10] Luisa C Lopez Cara, María Dora Carrión Peregrina, and María de la Encarnación Camacho Quesada. Utilización y aplicación de la herramienta "google docs" en la docencia universitaria dentro del marco del eees. In Univest 11, Gerona, 2011.

[11] Miguel Álvarez and Luis Sánchez Cañizares. Conocimiento, valoración y utilización, por parte del alumnado, de Google Drive como herramienta de trabajo cooperativo. Enseñanza $\&$ Teaching: Revista Interuniversitaria de Didáctica, 32(2):23-52, oct 2014.

[12] Daniel Rodrigo-Cano, Marcela IglesiasOnofrio, and Ignacio Aguaded. Metodologías participativas en la nube: La "g-Google" vs. la "Generación X" en la Web 2.0. Revista Complutense de Educacion, 28(1):223-237, 2017.

[13] M C Tolosa Bailén and J R García Bernabeu. "Google sites çomo herramienta educativa. In Jornadas de Redes de Investigación en Docencia Universitaria, page 1, 2011.

[14] Lawrence J. Awuah. Supporting 21stCentury Teaching and Learning: The Role of Google Apps for Education (GAFE). Journal of Instructional Research, 4, 2015.

[15] Maria Lindh, Jan Nolin, and Karen Nowé Hedvall. Pupils in the clouds: Implementation of Google Apps for Education. First Monday, 21(4), mar 2016.

[16] Maury Elizabeth Brown and Daniel L. Hocutt. Learning to Use, Useful for Learning: A Usability Study of Google Apps for Education. Journal of Usability Studies, 10(4):160$181,2015$.

[17] Keith R Heggart and Joanne Yoo. Getting the Most from Google Classroom: A Pedagogical Framework for Tertiary Educators. Australian Journal of Teacher Education, 43(433), 2018.

[18] María del Mar Moya Fuentes, Alejandro Bia Platas, María del Mar Carrasco Andrino, Almudena Jiménez Pascual, Aitana Ramón Martín, and Carolina Soler García. Memoria Red en metodologías docentes con TICS 2016/2017: implementación de la plataforma virtual Google Classroom. Universidad de Alicante. Instituto de Ciencias de la Educación, 2017.

[19] Natasha Singer. How Google took over the classroom. The New York Times, (May 13):1-15, 2017.
[20] Borja Gonzalez. Las bases de Google Classroom - The Flipped Classroom.

[21] Borja Gonzalez. Guía de Google Classroom para estudiantes - The Flipped Classroom.

[22] Juan Carlos Guerra. Tutorial de Google Classroom - Crear una clase, 2017.

[23] Rosa Liarte. Tutorial completo de Google Classroom para profesores.

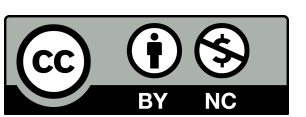

(C) 2018 by the authors. Submitted for possible open access publication under the terms and conditions of the Creative Commons Attribution CC-BY-NC 3.0 license (http://creativecommons.org/licenses/by-nc/3.0/). 